\title{
Diabetic Ketoacidosis Triggered by an Emphysematous Urinary Tract Infection: A Case Report and Mini-Review of Literature
}

\author{
Buenrostro-Valenzuela J C ${ }^{1}$, Amezquita-Perez J ${ }^{2}$, \\ Schlie-Villa $\mathbf{W}^{2}$ and Romero-Bermudez $\mathrm{J}^{1 *}$ \\ ${ }^{1}$ Department of Internal Medicine, Mexico's General \\ Hospital “Dr Eduardo Liceaga”, Mexico \\ ${ }^{2}$ Department of Cardiology, National Medical Center \\ "La Raza" Specialties' Hospital "Antonio Fraga Mouret" \\ (IMSS), Mexico \\ *Correspondling author: Romero-Bermudez J , \\ Department of Internal Medicine, Mexico's General \\ Hospital “Dr Eduardo Liceaga”, 148 Dr Balmis, Mexico
}

Received: May 25, 2021; Accepted: J une 23, 2021;

Published: June 30, 2021

\begin{abstract}
Generally, the most common triggers for Diabetic Ketoacidosis (DKA) are infectious diseases, such as Urinary Tract Infections (UTIs) or pneumonia. However, emphysematous infections are significant diseases rarely associated with DKA. Here, we present two cases of emphysematous urinary tract infection associated to diabetic ketoacidosis, highlighting the importance of a timely intervention and treatment. We review the need for appropriate laboratory and image testing in the context of infected patients who do not reach inflammatory/ glycemic goals to diagnosticate complicated infectious processes. This case report and mini-review also explore pathophysiology, the association of DKA and urinary emphysematous infections and treatment options.
\end{abstract}

Keywords: Diabetic ketoacidosis; Emphysematous pyelonephritis; Emphysematous cystitis

\section{Introduction}

Emphysematous pyelonephritis is an acute necrotizing infection of the urinary tract and/or adjacent tissues, in which gas accumulation occurs within the collecting system, renal parenchyma, or surrounding tissue, depending on severity [1-3].

A higher prevalence in the female gender has been described, as well as an average age presentation of 60 years [2].

The main risk factors associated in case series in literature are: type 2 diabetes mellitus, in up to $95 \%$ of patients with emphysematous pyelonephritis have preceding deficient glycemic control, followed by urinary tract obstruction $[2,3]$.

Most type 2 diabetes patients exhibit inadequate glycemic control levels upon admission confirmed by HbA1C levels greater than $8 \%$ or presence of microvascular complications (diabetic retinopathy/nephropathy) [2]. The pairing of diabetic ketoacidosis and emphysematous pyelonephritis is uncommon, this correlation is rare and few cases have been documented, being an important mortality outcome predictor $[4,5]$. In these cases, clinical or surgical treatment evidence is still lacking. However, successful conservative management has been reported despite metabolic decompensation in HUANG stages 2 or lower [6].

Among pathogenic agents, Escherichia coli is the most commonly cultured organism cultured, followed by Klebsiella pneumonia [2]. Clinical presentation is characterized by fever accompanied with abdominal pain (predominantly in flanks or lumbar region) as the most frequent symptoms, and may also be accompanied by nausea or vomiting [3].

Computed Tomography (CT) scan remains the modality of choice to demonstrate the presence of gas, distribution and extent of the disease. Making it the perfect image study to adequately classify this complicated infection. Furthermore, this test has treatment and prognosis implications [3].

Regarding the management of emphysematous pyelonephritis, different authors have described patient benefit from adequate fluid resuscitation, electrolyte management, strict glycemic control, and broad-spectrum antibiotic administration. In the correlation of emphysematous pyelonephritis and diabetic ketoacidosis, it is important to take therapeutic actions to both infection and metabolic decompensation. This is accomplished with low insulin doses administered by continuous pump infusion as well as fluid therapy; antibiotic treatment should be modified culture results are available [6]. Satisfactory results have been reported with conservative medical treatment or medical treatment combined with percutaneous drainage in cases of localized emphysematous pyelonephritis (HUANG 1 or 2) $[2,6]$.

Here we present a case report of emphysematous pyelonephritis with concomitant diabetic ketoacidosis.

\section{Case Presentation}

\section{First case}

A 37-year-old type 2 poorly controlled diabetic woman with a history of multiple UTIs was admitted to Emergency Department. Upon admission, the patient referred two-week history of malaise with intermittent fever accompanied by poliaquiuria, followed by abdominal and lumbar pain. Initial evaluation revealed tachycardia, tachypnea, hypotension, signs of dehydration, and generalized abdominal pain with extension to the lumbar region, in addition to a positive bilateral Giordano's sign. Studies revealed leukocytosis plus neutrophilia, hyperglycemia, hyperazoemia and a decompensated high anion gap metabolic acidosis, meeting criteria for moderate DKA. Initial urinalysis reported positive leucose esterase, bacteria, glycosuria and ketonuria; which pointed out a possible infection
J Fam Med - Volume 8 Issue 5 - 2021

ISSN : 2380-0658 | www.austinpublishing group.com

Romero-Bermudez et al. @ All rights are reserved
Citation: Buenrostro-Valenzuela JC, Amezquita-Perez J, Schlie-Villa W and Romero-Bermudez J. Diabetic Ketoacidosis Triggered by an Emphysematous Urinary Tract Infection: A Case Report and Mini-Review of Literature. J Fam Med. 2021; 8(5): 1259. 


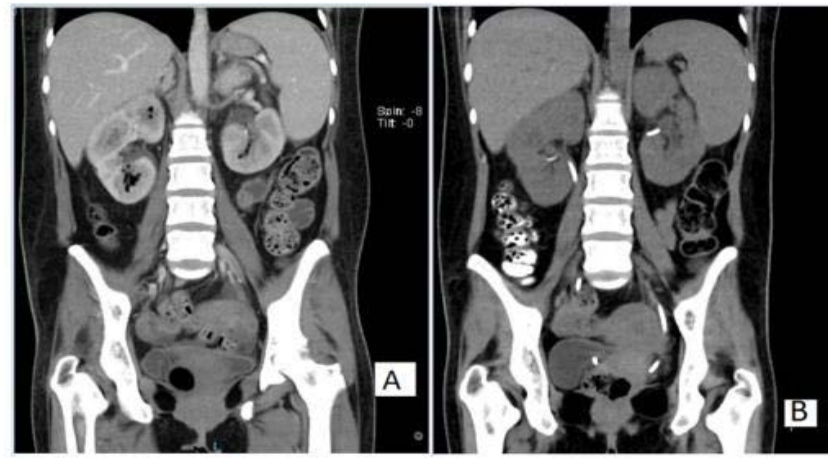

Figure 1: A) CT scan showing emphysematous pyelonephritis and cystitis. B) CT scan showing resolution emphysematous pyelonephritis and cystitis after medical treatment.

site, renal sonography revealed acute bilateral pyelonephritis. Initial management included intravenous fluids, empirical antibiotic therapy and insulin infusion. Despite this, abdominal pain and hyperglycemia persisted. Urine culture reported ESBL E. coli and antibiotic therapy was switched, we conducted an abdominal CT scan revealed emphysematous pyelonephritis Huang 2 and emphysematous cystitis (Figure 1A) and performed specific medical treatment. After 14 days, we performed a new abdominal CT scan with resolution of both emphysematous infections, DKA and was discharged home (Figure 1B).

\section{Second case}

A 50-year-old woman was admitted due to abdominal pain, nausea, vomiting and shortness of breath. She had a medical history of chronic kidney disease due to type 2 diabetes mellitus and hypertension. After admission abdominal pain exacerbated, and she became dehydrated. Physical examination and studies revealed tachycardia, tachypnea, hypotension, mild leukocytosis plus neutrophilia, hyperglycemia, hyperazoemia and a decompensated high anion gap metabolic acidosis, meeting criteria for severe DKA and septic shock. Initial urinalysis showed uncountable leukocytes and bacteria, glycosuria, ketonuria; which pointed out a possible infection site. Initial management include rehydration with crystalloids, insulin pump and empirical antibiotic therapy. The patient breathing returned to normal and achieved glucose control, but fever and abdominal pain persisted. We performed abdominal CT scan that showed right emphysematous pyelonephritis Huang 2 and emphysematous cystitis (Figure 2A) and performed specific medical treatment. After 14 days, we performed a new abdominal CT scan with resolution of infectious process (Figure 2B).

\section{Discussion}

Emphysematous pyelonephritis is an acute necrotizing infection of the renal parenchyma and adjacent tissues, in which gas accumulation occurs in the collecting system, renal parenchyma, or surrounding tissue, depending on severity [1-3]. Huang classified this entity into 5 categories that have prognostic value based upon CT scan findings: class 1 is characterized by gas in the collecting system only; class 2 characterized by gas in the renal parenchyma without extension to the extrarenal space; class $3 \mathrm{~A}$ characterized by extension of gas or abscess to the perinephric space; class $3 \mathrm{~B}$ characterized
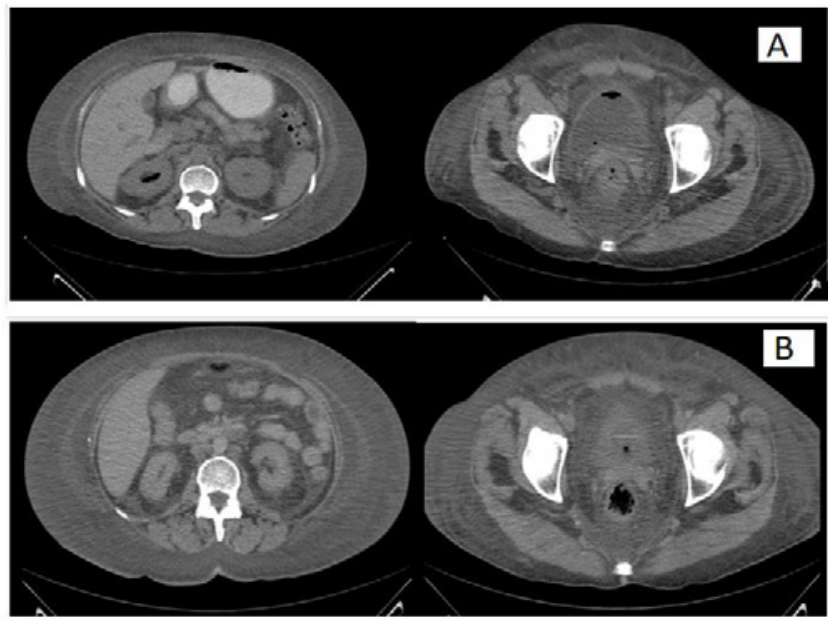

Figure 2: A) CT scan showing emphysematous pyelonephritis (right) and cystitis (left). B) CT scan showing resolution of emphysematous pyelonephritis (right) and cystitis (left).

by extension of gas or abscess to the pararenal space; and class 4 characterized by bilateral emphysematous pyelonephritis or a solitary functioning kidney with emphysematous pyelonephritis [2].

A higher prevalence in the female gender has been described, as well as an average age presentation at 60 years. The main risk factors identified in case series in the literature are type 2 diabetes mellitus, up to $95 \%$ of patients with emphysematous pyelonephritis have poor glycemic control, followed by urinary tract obstruction $[2,4,7]$.

Most type 2 diabetes patients exhibit inadequate glycemic control levels upon admission confirmed by HbA1C levels greater than $8 \%$ or presence of microvascular complications (diabetic retinopathy/ nephropathy) $[7,8]$. One study reported that neutrophiles' ability to produce Neutrophil Extracellular Traps (NETs) was suppressed during hyperglycemia, leading to infection susceptibility; macrophage function is also altered. Another study demonstrated that chronic hyperglycemia was significantly associated with complement and crystallizable fragments of the $\operatorname{IgG}$ receptor deficit on isolated monocytes, resulting in phagocytosis impairment $[9,10]$.

It's known that DKA may be precipitated by the catabolic stress of acute illness such as trauma, surgery, or infections [11]. Diabetic ketoacidosis triggered by emphysematous pyelonephrtitis is uncommon, this correlation is rare, and few cases have been documented, being an important predictor of death [4-6]. In a cohort of 26 patients with emphysematous pyelonephritis, DKA has an incidence of $27 \%$ (7 patients) and it's associated with increased mortality rate [5]. Another comparative study showed that patients with EPN had commonly poor glycemic control, lower estimated glomerular filtration rate and complications like DKA compared with patients with pyelonephritis [7].

The most frequently isolated gas-producing organisms in urinary tract infections are Escherichia coli in $63 \%$. Nonetheless, others agents such as: Klebsiella pneumoniae, Proteus mirabilis, Pseudomonas aeruginosa, Enterobacter aerogenes, Enterococci, Staphylococcus aureus, Streptococci, Nocardia, Clostridium perfringens and Candida albicans are also usually involved [2,7]. 
The management of DKA is with intravenous fluids, insulin therapy, potassium and bicarbonate when necessary, but treatment of concomitant EPN is debated. Nowadays there are not international guidelines or established recommendations for EPN management and available information is poorly weighted given the low incidence reported of EPN [7,12].

Since 1980's treatment of patients with EPN was emergency nephrectomy and/or open surgical drainage together with antibiotic therapy, nowadays some studies suggest better outcomes with nonsurgical management like $>90 \%$ of survival and less decline in glomerular filtration rate $[13,14]$.

\section{Conclusion}

It is widely known that in type 2 diabetic patients, infectious processes are the most common decompensation causes. However, emphysematous infections are rarely seen triggering DKA and require immediate aggressive management.

The key to a favorable outcome in these patients were a timely specific intervention, guided antibiotic therapy and glycemic control. Lack of initial achievement of inflammatory/glycemic goals should prompt physicians to look out for this deadly pair.

\section{References}

1. Ubee SS, McGlynn L, Fordham M. Emphysematous pyelonephritis. BJU Int 2011; 107:1474-1478.

2. Huang JJ, Tseng CC. Emphysematous pyelonephritis: clinicoradiological classification, management, prognosis, and pathogenesis. Arch Intern Med. 2000; 160: 797-805.

3. Sokhal AK, Kumar M, Purkait B, Jhanwar A, Singh K, Bansal A, et al Emphysematous pyelonephritis: Changing trend of clinical spectrum, pathogenesis, management and outcome. Turk J Urol. 2017; 43: 202-209.

4. Falagas ME, Alexiou VG, Giannopoulou KP, Siempos II. Risk factors for mortality in patients with emphysematous pyelonephritis: a meta-analysis. J Urol. 2007; 178: 880-885.
5. Wang JM, Lim HK, Pang KK. Emphysematous pyelonephritis. Scand J Urol Nephrol. 2007; 41: 223-229.

6. Song $Y$, Shen $X$. Diabetic ketoacidosis complicated by emphysematous pyelonephritis: a case report and literature review. BMC Urol. 2020; 20: 6.

7. Nabi T, Rafiq N, Rahman MHU, Rasool S, Wani NUD. Comparative study of emphysematous pyelonephritis and pyelonephritis in type 2 diabetes: a single-centre experience. J Diabetes Metab Disord. 2020; 19: 1273-1282.

8. Joshi MB, Lad A, Bharath Prasad AS, Balakrishnan A, Ramachandra L, Satyamoorthy K. High glucose modulates IL-6 mediated immune homeostasis through impeding neutrophil extracellular trap formation. FEBS Lett. 2013; 587: 2241-2246.

9. Restrepo BI, Twahirwa M, Rahbar MH, Schlesinger LS. Phagocytosis via complement or Fc-gamma receptors is compromised in monocytes from type 2 diabetes patients with chronic hyperglycemia. PLoS One. 2014; 9: e92977.

10. Berbudi A, Rahmadika N, Tjahjadi Al, Ruslami R. Type 2 Diabetes and its Impact on the Immune System. Curr Diabetes Rev. 2020; 16: 442-449.

11. Rashid MO, Sheikh A, Salam A, Farooq S, Kiran Z, Islam N. Diabetic ketoacidosis characteristics and differences In type 1 versus type 2 diabetes patients. J Ayub Med Coll Abbottabad. 2017; 29: 398-402.

12. Maletkovic J, Isorena JP, Palma Diaz MF, Korenman SG, Yeh MW. Multifactorial hypercalcemia and literature review on primary hyperparathyroidism associated with lymphoma. Case Rep Endocrinol. 2014; 2014: 893134.

13. Somani BK, Nabi G, Thorpe P, Hussey J, Cook J, N'Dow J, et al. Is percutaneous drainage the new gold standard in the management of emphysematous pyelonephritis? Evidence from a systematic review. J Urol. 2008; 179: 1844-1849.

14. Elawdy MM, Osman Y, Abouelkheir RT, El-Halwagy S, Awad B, El-Mekresh M. Emphysematous pyelonephritis treatment strategies in correlation to the CT classification: have the current experience and prognosis changed? Int Urol Nephrol. 2019; 51: 1709-1713. 